\title{
The "round trip" theory for reconstruction of Green's functions at passive locations
}

\author{
Andy Moorhouse ${ }^{\text {a) }}$ and Andy Elliott \\ Acoustics Research Centre, University of Salford, Salford, Greater Manchester, M5 4WT, United Kingdom
}

(Received 13 March 2013; revised 24 August 2013; accepted 28 August 2013)

\begin{abstract}
An expression for the Green's function at an arbitrary set of passive locations (no applied force) is derived and validated by experiment. Three sets of points are involved, the passive reconstruction points, $\mathbf{c}$, which lie on a virtual boundary and two sets of auxiliary points, denoted $\mathbf{a}$ and $\mathbf{b}$, located either side. The reconstruction is achieved using Green's functions forming a "round trip" from and to the reconstruction points via $\mathbf{a}$ and $\mathbf{b}$. A two stage measurement procedure is described involving excitation at $\mathbf{b}$ and $\mathbf{a}$ but with no excitation required at the reconstruction points. A known "round trip" relationship is first introduced which is theoretically exact for points on a multi-point interface between two linear, time invariant subsystems. Experimental results for frequency response functions of a beam-plate structure show that this relationship gives good results in practice. It is then shown that the theory provides an Nth order approximation for the Green's function at arbitrary points, where $\mathrm{N}$ is the number of points at $\mathbf{b}$. The expression is validated by reconstructing point and transfer frequency response functions at two passive points on an aluminum plate.
\end{abstract}

(C) 2013 Acoustical Society of America. [http://dx.doi.org/10.1121/1.4821210]

PACS number(s): 43.40.At, 43.20.Bi, 43.40.Sk, 43.58.Bh [KML]

Pages: $3605-3612$

\section{INTRODUCTION}

The problem addressed in this paper is that of obtaining Green's functions at passive points, where the term "passive" indicates that no external force is applied. The practical application is in the determination of Green's functions at locations where the structure cannot be excited, for example due to lack of access.

In the structural dynamics field, several authors have investigated the possibility of constructing an $\mathrm{M} \times \mathrm{M}$ frequency response function (FRF) matrix without applying a force at every point. (Note that in mechanical engineering applications the term "FRF" tends to be used rather than Green's function). Ashory et al. ${ }^{1,2}$ and Silva et al. ${ }^{3}$ have presented methods in which the entire $\mathrm{M} \times \mathrm{M}$ FRF matrix can theoretically be constructed from just one column, i.e., requiring response measurement at all $\mathrm{M}$ locations but excitation at just one of these positions. Both methods exploit the fact that accelerometers of known finite mass will load the structure by a calculable amount. Therefore, by performing repeated tests with varying mass loading a sufficient number of equations can be constructed which can be solved for the FRFs at the passive locations. The reconstructed FRFs are however sensitive to measurement errors, particularly if the added masses produce only a small change in the FRFs. Ewins' approach ${ }^{4}$ also allows reconstruction of the entire matrix from a single column and does not require repeated tests. However, mode frequencies and mode shapes must be extracted from $\mathrm{M}$ responses to a single excitation and this need for a modal decomposition makes the approach unsuitable for structures with many modes.

\footnotetext{
a) Author to whom correspondence should be addressed. Electronic mail: a.t.moorhouse@salford.ac.uk
}

In structural dynamic applications, such as those mentioned above, it is often practicable to apply controlled excitation of the test structure, albeit not always at the points of interest or in the desired directions. Research has also been ongoing across several other disciplines where different practical problems are encountered, for example, in civil engineering where artificial excitation is problematic and exploitation of naturally occurring excitation offers some advantages. ${ }^{5}$ Two main techniques have emerged for retrieval of Green's functions (the term commonly used in various fields): Time reversal (e.g., Ref. 6) and correlation (e.g., Refs. 5 and 7). A comprehensive review of these techniques is beyond the scope of this paper and the reader is referred to reviews by Larose et al. ${ }^{8}$ and more recently by Margerin and Sato $^{9}$ for more details. However, within this literature is a result of particular relevance to this paper, namely, Draeger and Fink's "cavity equation." 6 The basic form of their equation relates responses at one source and one receiver point inside a chaotic cavity. The authors show that a timereversed signal, i.e., one that is captured at the receiver, time-reversed, and retransmitted back to the source, can be expressed in terms of a convolution of the impulse response functions of the receiver and source points (the latter also being time-reversed). In order to reach this result the assumption of no degenerate modes was invoked, which allows the responses to be expressed as a modal sum without cross coupling between modes. The equation was shown to work well for irregular cavities ${ }^{10}$ (where a lack of degenerate modes would be expected) but started to break down for regular enclosures. The cavity equation has been picked up by several authors, for example, by Derode et al. ${ }^{11}$ who extended the work to open media for which an array of sensors was required. However, of most direct interest to the theory presented in this paper is a form of the cavity equation ${ }^{6}$ which is generalized so as to include a third 
(observation) point. This form will be seen to bear some resemblance with the results to be derived in the following section and a discussion of the similarities and differences will be deferred until then.

The following section provides a theoretical development, starting with a known result from Moorhouse et al. ${ }^{12}$ in which an exact "round trip" relationship was derived for passive points on a multi-point interface between two substructures. A new derivation is offered in Sec. II A followed by an experimental validation in Sec. II B. In Sec. III the application of the round trip theory to Green's functions at arbitrary passive points is considered. Note that while much of the following is presented in terms of structural systems, the theory applies to linear, time invariant systems generally. We use the term frequency response function (FRF) where fixed excitation or response points are involved and "Green's function" for variable points, although this distinction is not always clear cut.

\section{DISCREET INTERFACES}

\section{A. Theory}

Consider a linear, time-invariant (LTI) structure in which two domains, A and B, are separated by a multi-point interface (see Fig. 1). Points a and b lie, respectively, in domains A, B and points $\mathbf{c}$ lie on the interface. We define the FRF matrices between the various sets of points as, e.g., $\mathbf{Y}_{\mathbf{a b}}$ where $\mathbf{a}, \mathbf{b}$ are the response and excitation positions, respectively. In Ref. 12, a relationship was derived in which the matrix of FRFs on the interface, $\mathbf{Y}_{\mathbf{c c}}$, was expressed in terms of three other FRF matrices. In the following, an alternative derivation is given.

Let the assembly be excited by a single point force at an arbitrary position $b_{i}$. Assuming harmonic excitation the responses at positions $\mathbf{a}$ and $\mathbf{c}$ are given by

$$
\begin{aligned}
& \mathbf{v}_{\mathbf{a}}^{\left(\mathbf{b}_{\mathbf{i}}\right)}=\mathbf{Y}_{\mathbf{a b}} f_{b_{i}}, \\
& \mathbf{v}_{\mathbf{c}}^{\left(\mathbf{b}_{\mathbf{i}}\right)}=\mathbf{Y}_{\mathrm{cb}} f_{b_{i}},
\end{aligned}
$$

where $\boldsymbol{f}_{\boldsymbol{b}_{\boldsymbol{i}}}=\left\{0, \ldots, f_{b_{i}}, 0, \ldots 0\right\}^{\boldsymbol{T}}$ is the vector of forces applied at $\mathbf{b}$ and the superscript $\left(b_{i}\right)$ indicates excitation at $b_{i}$. We now wish to generate an identical velocity field in substructure A but by applying a set of forces at the interface $\mathbf{c}$ rather than at $b_{i}$. Bobrovnitskii ${ }^{13}$ (see also Ref. 14) has shown that this occurs when the forces applied at $\mathbf{c}$ are equal and opposite to the "blocked forces," denoted $\mathbf{f}_{\mathbf{b l}}^{\left(\mathbf{b}_{\mathbf{i}}\right)}$, i.e., the reaction forces obtained at $\mathbf{c}$ under the action of $\boldsymbol{f}_{b_{i}}$ when $\mathrm{c}$ is blocked. Thus, the forces $-\mathbf{f}_{\mathbf{b l}}^{\left(\mathbf{b}_{\mathbf{i}}\right)}$ at $\mathbf{c}$ and $\boldsymbol{f}_{\boldsymbol{b}_{\boldsymbol{i}}}$ at $b_{i}$ are equivalent in the sense that they generate an identical velocity field in substructure A. Using this new equivalent excitation, the velocity at the same points as before can be re-expressed in terms of the blocked forces

$$
\begin{aligned}
& \mathbf{v}_{\mathbf{a}}^{\left(\mathbf{b}_{\mathbf{i}}\right)}=-\mathbf{Y}_{\mathbf{a c}} \mathbf{f}_{\mathbf{b l}}^{\left(\mathbf{b}_{\mathbf{i}}\right)}, \\
& \mathbf{v}_{\mathbf{c}}^{\left(\mathbf{b}_{\mathbf{i}}\right)}=-\mathbf{Y}_{\mathbf{c c}} \mathbf{f}_{\mathbf{b l}}^{\left(\mathbf{b}_{\mathbf{i}}\right)} .
\end{aligned}
$$

Eliminating the forces from Eq. (1) and (2) we obtain (a)

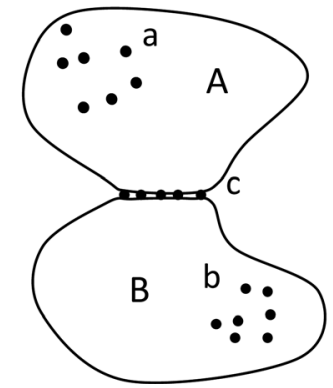

(b)

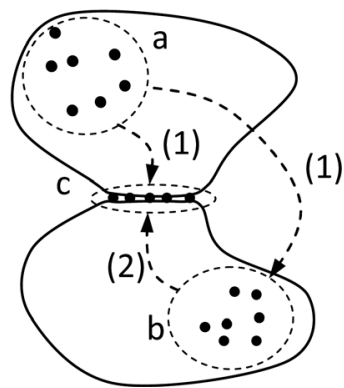

FIG. 1. (a) Schematic of two substructures, A and B, connected at an interface c. (b) Schematic indication of the "round trip" path when $\mathbf{c}$ are passive points.

$$
\mathbf{v}_{\mathbf{c}}^{\left(\mathbf{b}_{\mathbf{i}}\right)}=\mathbf{Y}_{\mathbf{c b}} \mathbf{Y}_{\mathbf{a b}}^{-1} \mathbf{v}_{\mathbf{a}}^{\left(b_{i}\right)}
$$

where the inverse is assumed to exist and may be interpreted as a pseudo-inverse: A condition for a unique solution is $n_{a} \geq n_{b}$ where $n_{a}, n_{b}$ are the number of points, or more generally degrees of freedom, at $\mathbf{a}$ and $\mathbf{b}$, respectively. Equation (5) can be interpreted as being equivalent to a multi-channel deconvolution of the response at a. Introducing Eq. (3) into the right hand side and Eq. (4) into the left we get

$$
\mathbf{Y}_{\mathbf{c c}} \mathbf{f}_{\mathbf{b l}}^{\left(\mathbf{b}_{\mathbf{i}}\right)}=\mathbf{Y}_{\mathrm{cb}} \mathbf{Y}_{\mathbf{a b}}^{-1} \mathbf{Y}_{\mathbf{a c}} \mathbf{f}_{\mathrm{bl}}^{\left(\mathbf{b}_{\mathbf{i}}\right)}
$$

Equation (6) is an identity between two vectors. We may build up a set of such relationships by applying point forces at other points on $\mathbf{b}$ in turn and arranging the results into the columns of a matrix so as to arrive at

$$
\mathbf{Y}_{\mathrm{cc}} \mathbf{F}_{\mathrm{bl}}=\mathbf{Y}_{\mathrm{cb}} \mathbf{Y}_{\mathrm{ab}}^{-1} \mathbf{Y}_{\mathrm{ac}} \mathbf{F}_{\mathrm{bl}}
$$

where the columns of $\mathbf{F}_{\mathbf{b l}}=\left\{\mathbf{f}_{\mathbf{b l}}^{\left(\mathbf{b}_{\mathbf{l}}\right)} \mathbf{f}_{\mathbf{b l}}^{\left(\mathbf{b}_{2}\right)} \cdots \mathbf{f}_{\mathbf{b l}}^{\left(\mathbf{b}_{\mathbf{b}}\right)}\right\}_{n_{c} \times n_{b}}$ are the blocked force vectors corresponding to excitation at each position on $\mathbf{b}$. If the inverse of $\mathbf{F}_{\mathbf{b l}}$ exists then both sides of Eq. (7) can be post-multiplied by its inverse (or pseudo inverse) to yield an identity between the matrices

$$
\mathbf{Y}_{\mathrm{cc}}=\mathbf{Y}_{\mathrm{cb}} \mathbf{Y}_{\mathrm{ab}}^{-1} \mathbf{Y}_{\mathrm{ac}}
$$

A condition for uniqueness is $n_{b} \geq n_{c}$. Equation (8) is one form of the round trip relationship. A more advantageous form can be obtained by using the substitutions $\mathbf{Y}_{\mathbf{a c}}=\mathbf{Y}_{\mathbf{c a}}^{\mathbf{T}}$, $\mathbf{Y}_{\mathbf{a b}}=\mathbf{Y}_{\mathrm{ba}}^{\mathbf{T}}$ and noting that $\mathbf{Y}_{\mathbf{c c}}=\mathbf{Y}_{\mathrm{cc}}^{\mathbf{T}}$, giving

$$
\mathbf{Y}_{\mathrm{cc}}=\mathbf{Y}_{\mathrm{ca}} \mathbf{Y}_{\mathrm{ba}}^{-1} \mathbf{Y}_{\mathrm{cb}}^{\mathrm{T}}
$$

Equation (9) is the same as that given in Ref. 12. It expresses the FRF on the interface points, $\mathbf{c}$, in terms of three other sets of FRFs. Looking at the indices on the right hand side we see that the three matrices describe the three legs of a round trip journey, from $\mathbf{c}$ to $\mathbf{a}, \mathbf{a}$ to $\mathbf{b}$, and $\mathbf{b}$ to $\mathbf{c}$. Hence this relationship has become known as the "round trip theory."15 In Eq. (9) these paths are oriented as shown in Fig. 1(b), and describe a round trip $\mathbf{c}-\mathbf{a}-\mathbf{b}-\mathbf{c}$ with the first leg reversed. This orientation turns out to be advantageous, in that no 
excitation is then required at c, i.e., these points are passive points. Thus, Eq. (9) provides an expression for obtaining FRFs at a set of passive points.

For completeness, we observe that the substructures could be interchanged so that Eq. (9) has a dual form in which the direction of all paths is reversed. Moreover, it is clear that Eq. (9) may be rearranged so as to express any of the FRF matrices in terms of the three other legs of the round trip although here we focus here on the "point" FRF (same response and excitation points) whose practical applications are most obvious.

Noting that points a are "excitation-only" points and that points b are "excitation and response" points, a twostage measurement is convenient to describe the three elements of the round trip. In the first stage, the structure is excited at $\mathbf{a}$ and the response measured simultaneously at $\mathbf{b}$ and $\mathbf{c}$ [the paths marked (1) in Fig. 1]. Since the product of the first two terms on the rhs of Eq. (9) is equal to the generalized transmissibility $\left(\mathbf{T}=\mathbf{Y}_{\mathbf{c a}} \mathbf{Y}_{\mathbf{b a}}^{-\mathbf{1}}\right)$ (Ref. 16), this can be obtained from matrices of the responses at $\mathbf{a}$ and $\mathbf{c}$ under a set of different force distributions, without explicit knowledge of the applied forces. ${ }^{17}$ Thus, the first stage of the measurement can potentially use excitation from unknown forces, including naturally occurring sources or operational forces, although this possibility will not be further explored in this paper. The second stage measurement requires excitation with known forces at $\mathbf{b}$ and measurement of response at c to give the last leg of the round trip [the path marked (2) in Fig. 1]. In practice these measurements would often be done with a force hammer for structural systems or a volume velocity source for acoustic systems.

At this point we compare Eq. (9) with Draeger and Fink's generalized cavity equation, which (using their original notation) is given by ${ }^{6}$

$$
h_{\mathrm{AB}}(-t) * h_{\mathrm{BC}}(t)=h_{\mathrm{AC}}(t) * h_{\mathrm{BB}}(-t)
$$

in which $h_{\mathrm{AB}}(t)$ is the impulse response function, i.e., the response at location $\mathrm{B}$ due to a Dirac impulse at $\mathrm{A}$, etc., * represents convolution and $t$ time so that $(-t)$ implies a timereversed impulse response function. This form of the cavity equation ${ }^{6}$ is outwardly similar to Eq. (9) since it can be seen that the locations form a similar round trip. However, there are significant differences between the two formulations. The fact that Eq. (9) and Eq. (10) are defined, respectively, in the frequency and time domain is not by itself significant, however the cavity equation employs a time reversal, equivalent to a complex conjugate in the frequency domain, which is a fundamentally different operation to the matrix inversion of Eq. (9), equivalent to a multi-channel deconvolution in the time domain. Second, the cavity equation was derived for single excitation and response points anywhere in the cavity whereas Eq. (9) applies to multiple locations on the interface between substructures. A further difference is that the assumption of no degenerate modes is central to Draeger and Fink's derivation, ${ }^{6}$ but no such assumption was needed leading to Eq. (9) which is theoretically exact for LTI systems provided $n_{a} \geq n_{b} \geq n_{c}$.

\section{B. Experimental validation}

The round trip theory as presented above is exact in theory, and Moorhouse et al. ${ }^{12}$ demonstrated through simulations of rods and beams that, given exact input data, the FRFs at an internal interface are predicted exactly by Eq. (9). However, exact data are not available from measurements so experimental validation using a physical structure is needed. The test structure, illustrated in Fig. 2, consisted of a steel beam connected via two steel blocks to a $12.7 \mathrm{~mm}$ thick PVC plate. Twelve excitation-response positions were approximately evenly spaced on the beam (points b) and 80 hammer hits were made at random locations on the plate (points a). A total of 12 accelerometers were employed on

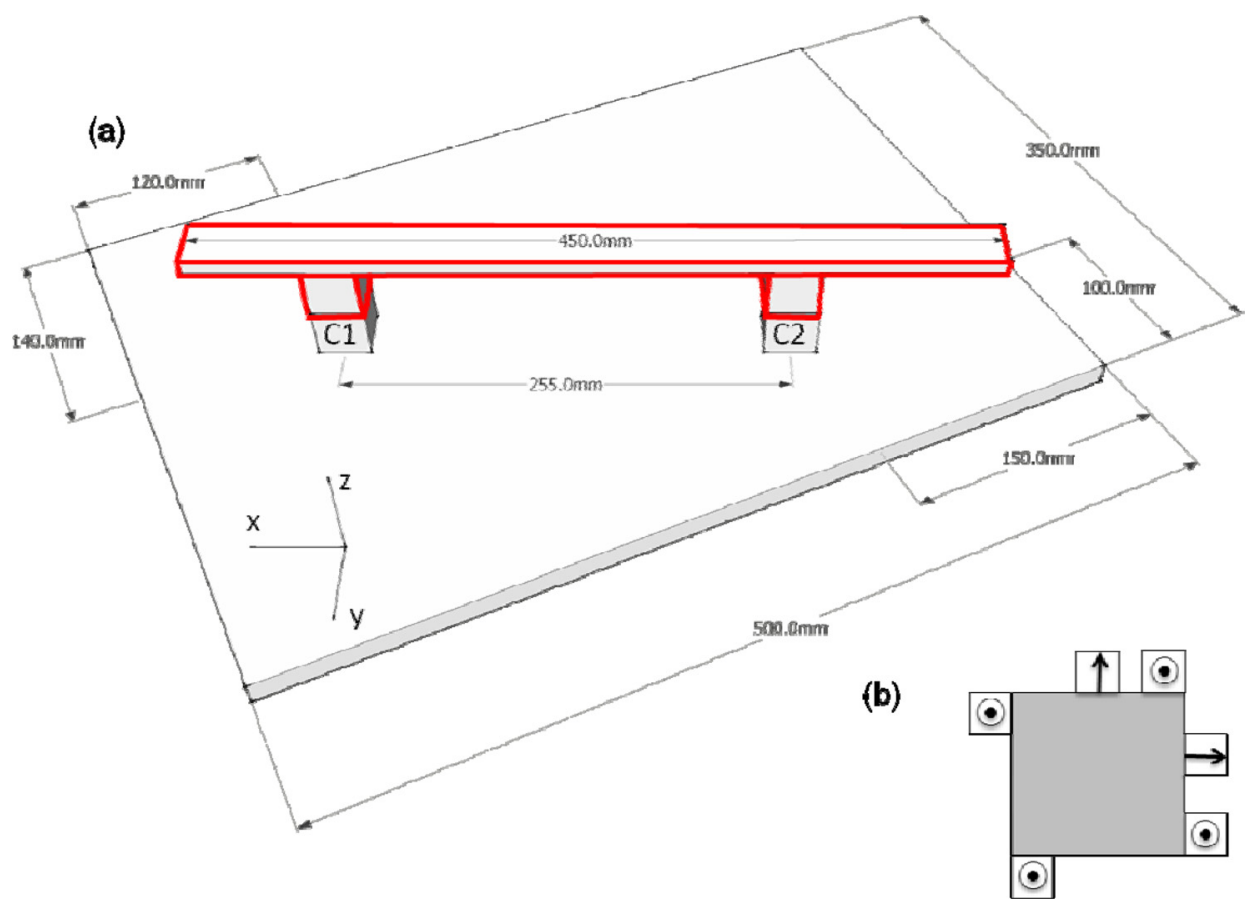

FIG. 2. (Color online) The beam-plate structure employed to test the discreet interface theory. (a) General assembly (b) detail showing accelerometer locations at the base of the feet. 
the interface (points c) as detailed in Fig. 2(b); pairs of sensors were used on either side of the block so that rotations could be inferred from the differences of the signals. ${ }^{18}$ The structure was excited with a force hammer with a plastic tip which gave a flat force spectrum, rolling off from around $500 \mathrm{~Hz}$ but with sufficient energy up to $2.5 \mathrm{kHz}$. The $12 \times$ 12 FRF matrix at the interface was then constructed using Eq. (9).

Physically, we expect five degrees of freedom at each of the two points forming the interface: $\mathrm{x}, \mathrm{y}, \mathrm{z}$ forces together with moments about the horizontal axes giving a total of ten degrees of freedom for the interface (moments about the vertical axis were considered negligible). The expected rank of the calculated $12 \times 12$ FRF matrix is therefore 10 so the solution was regularized by discarding two singular values at each frequency. ${ }^{19}$ Note that the structure was designed ${ }^{15}$ so that the $10 \times 10$ Green's function matrix for the interface could be measured directly for comparison with the indirect round-trip measurement although in many practical applications the direct measurement would not be possible.

The round trip estimates are compared with direct measurement for the vertical interface FRF in Fig. 3. Good agreement is evident over a wide frequency range. The small errors at low frequency are probably due to noise, emphasized by the small differences in the signals of the accelerometer pairs designed to capture rotations. Those at high frequencies are probably due to small (unintended) offsets between forcing and response points at a or $\mathbf{c}$. This confirms that the round trip approach can be applied successfully in practice.

Some interesting observations can be made from Fig. 3. First, note that the two transfer mobilities, $\mathrm{Y}_{12}$ and $\mathrm{Y}_{21}$ (ratio of velocity to force) given in Figs. 3(b) and 3(c) are theoretically identical by reciprocity. However the round trip estimates take different routes from point 1 to 2 , so different data is employed in each case and slightly differing estimates result. Second, it is interesting that a point mobility, which is a minimum phase function, can be successfully reconstructed from a set of transfer mobilities which are nonminimum phase functions: note that the phase of $\mathrm{Y}_{11}$ and $\mathrm{Y}_{22}$ lies between $\pm \pi$ indicating a positive real part [Figs. 3(e) and 3(h)] whereas the transfer mobilities forming the round trip have no such restriction on their phase.

\section{CONTINUOUS INTERFACES}

In this section we consider the application of the round trip theory, shown above to be exact for points on multipoint interfaces, to arbitrary points on a structure.

\section{A. Application to a subset of points on an interface}

Consider the case where indirect determination of FRFs is required at a subset of points, or degrees of freedom, on the interface. Thus, we partition the interface $\mathbf{c}$ into two
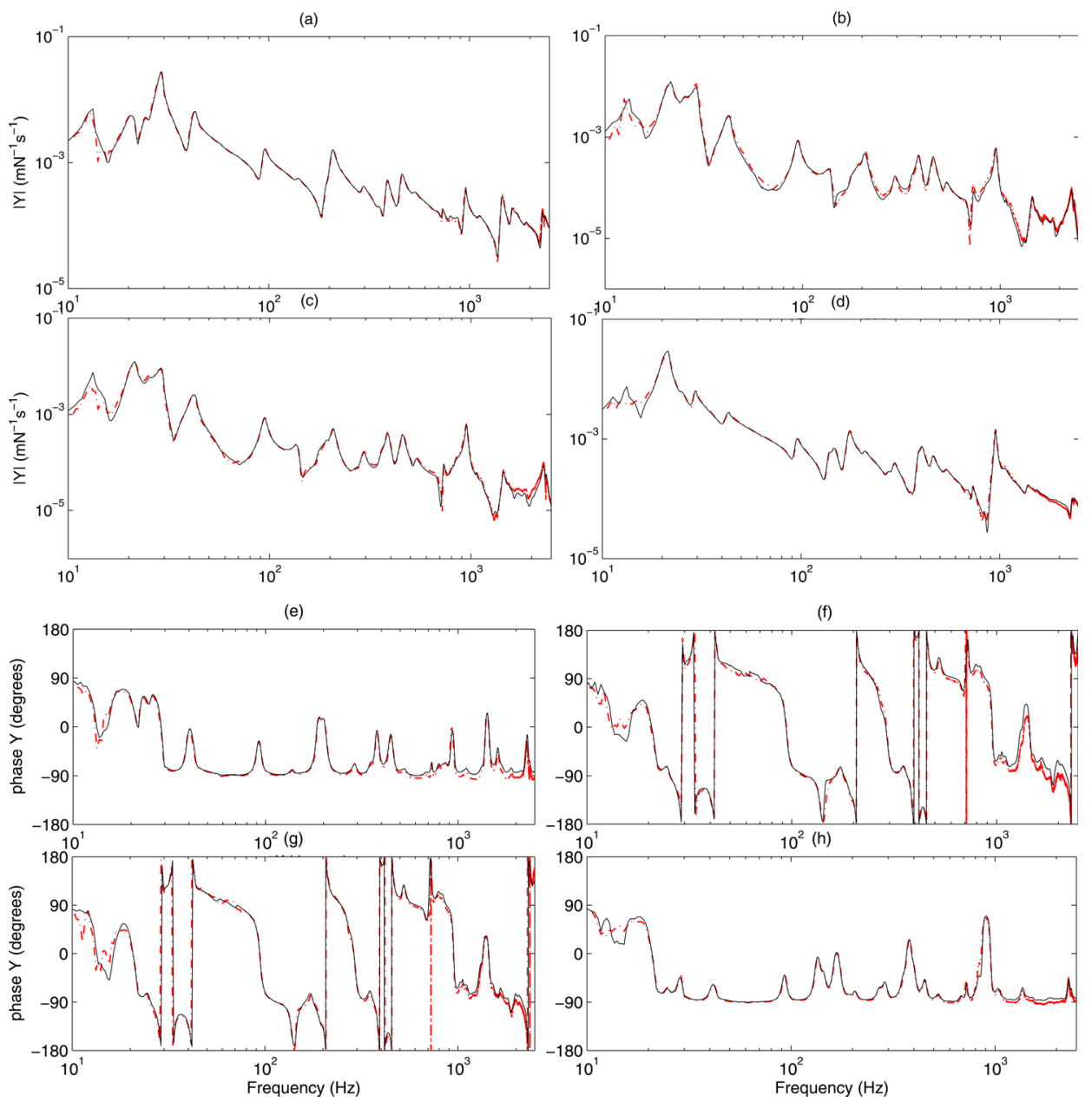

FIG. 3. (Color online) Comparison of directly measured mobility (continuous line) with round trip reconstruction (dotted line) in the out-of-plane (z) direction, magnitude and phase for the beam-plate structure. (a), (e) Point mobility at connection $1, \mathrm{Y}_{11}$ (b), (f) transfer mobility, $\mathrm{Y}_{12}$. (c), (g) $\mathrm{Y}_{21}$. (d), (h) point mobility at connection $2, \mathrm{Y}_{22}$. 
subsets: $\mathbf{c}_{1}$, at which indirect determination of the $\mathrm{FRF}(\mathrm{s})$ is required and $\mathbf{c}_{2}$, which comprises all remaining interface degrees of freedom. Eq. (9) can then be expressed as

$$
\left[\begin{array}{ll}
\mathbf{Y}_{\mathbf{c}_{1} \mathbf{c}_{1}} & \mathbf{Y}_{\mathbf{c}_{1} \mathbf{c}_{2}} \\
\mathbf{Y}_{\mathbf{c}_{2} \mathbf{c}_{1}} & \mathbf{Y}_{\mathbf{c}_{2} \mathbf{c}_{2}}
\end{array}\right]=\left[\begin{array}{l}
\mathbf{Y}_{\mathbf{c}_{1} \mathbf{a}} \\
\mathbf{Y}_{\mathbf{c}_{2} \mathbf{a}}
\end{array}\right] \mathbf{Y}_{\mathbf{b a}}^{-1}\left[\begin{array}{ll}
\mathbf{Y}_{\mathbf{c}_{1} \mathbf{b}}^{\mathbf{T}} & \mathbf{Y}_{\mathbf{c}_{2} \mathbf{b}}^{\mathbf{T}}
\end{array}\right]
$$

Since our particular interest is in subset $\mathbf{c}_{1}$, the required FRF matrix is the upper diagonal element

$$
\mathbf{Y}_{\mathbf{c}_{1} \mathbf{c}_{1}}=\mathbf{Y}_{\mathbf{c}_{1} \mathbf{a}} \mathbf{Y}_{\mathbf{b a}}^{-1} \mathbf{Y}_{\mathbf{c}_{1} \mathbf{b}}^{\mathbf{T}}
$$

Equation (12) has some interesting implications which will now be considered. First, we recall that the general round trip relation, Eq. (9), provides an exact expression for all FRF(s) on the interface between two substructures. Equation (12) shows that a similar round trip relation applies to an arbitrary subset of these degrees of freedom and, since no assumptions have been introduced along the way, this relationship is therefore also exact. However, despite the fact that Eq. (9) was derived in terms of the entire force distribution on the interface, explicit knowledge is required only of degrees of freedom in subset $\mathbf{c}_{1}$. The remaining degrees of freedom on the interface, $\mathbf{c}_{2}$, do not appear explicitly in Eq. (12) although their influence is included in a very general sense in that the condition $n_{a} \geq n_{b} \geq n_{c}=n_{c 1}+n_{c 2}$ (see Sec. II) must still be met. In other words, it is sufficient to account for the number of degrees of freedom in $\mathbf{c}_{2}$ without any other knowledge about their role or nature.

This suggests that the choice of the interface is arbitrary provided that it includes the reconstruction points $\mathbf{c}_{1}$, and that the total number of degrees of freedom along the interface does not exceed the number of points at $\mathbf{a}$ and $\mathbf{b}$. If this is so, then the sub-structuring into A and B is also arbitrary and the question arises as to whether Eq. (12) can be used to obtain Green's functions at arbitrary points on a structure (see Fig. 4). To address this question, we need to consider the application of the round trip theory for continuous interfaces which is addressed in the following.

\section{B. Extension to continuous interfaces and arbitrary sub-domains}

Consider the structure shown in Fig. 4 separated into two domains by a continuous virtual boundary, $s$, which passes through the reconstruction points, $\mathbf{c}$, but is otherwise arbitrary. This arrangement is similar to that in Fig. 1 except that the $\mathbf{c}$ points form only a subset of the boundary which is now continuous. Note that this implies that the "substructures" located either side of the boundary need not correspond to physically separable substructures. Since the boundary is continuous we suppose that an infinite number of degrees of freedom will be required to represent the action of one "substructure" on the other. Thus, while in principle Eq. (12) might apply, in practice an infinite number of points would be required either side for an exact reconstruction of the Green's functions at points on $s$. The question then arises as to whether an approximate reconstruction can be achieved with a finite set of points.
The derivation follows essentially the same steps as in Sec. II A which led to the round trip relationship [Eq. (8)]. The first step is to consider excitation with a point force $f_{b_{i}}$ at point $b_{i}$ (Fig. 4). The velocity at points a and on the interface is then given by

$$
\begin{aligned}
& \mathbf{v}_{\mathbf{a}}^{\left(b_{i}\right)}=\mathbf{Y}_{\mathrm{ab}} \boldsymbol{f}_{\boldsymbol{b}_{i}}, \\
& v(s)^{\left(b_{i}\right)}=g\left(s \mid \boldsymbol{b}^{\boldsymbol{T}}\right) \boldsymbol{f}_{\boldsymbol{b}_{i}},
\end{aligned}
$$

where $v(s)^{\left(b_{i}\right)}$ is the continuous velocity distribution on the interface and $g\left(s \mid \boldsymbol{b}^{\boldsymbol{T}}\right)=\left\{g\left(s \mid b_{1}\right) g\left(s \mid b_{2}\right) \cdots g\left(s \mid b_{n_{b}}\right)\right\}$ is a row vector of Green's functions joining the interface to points on b. As before we replace the excitation at $b_{i}$ with the equivalent excitation, i.e., the blocked force distribution $f(s)^{\left(b_{i}\right)}$ applied to the interface. ${ }^{13,14}$ The velocity at the same points as before can thus be re-expressed in terms of an integration over the interface

$$
\begin{aligned}
& \mathbf{v}_{\mathbf{a}}=\int_{s^{\prime}} g\left(\mathbf{a} \mid s^{\prime}\right) f\left(s^{\prime}\right)^{\left(b_{i}\right)} d s^{\prime}, \\
& v(s)^{\left(\boldsymbol{b}_{i}\right)}=\int_{s^{\prime}} g\left(s \mid s^{\prime}\right) f\left(s^{\prime}\right)^{\left(b_{i}\right)} d s^{\prime},
\end{aligned}
$$

where $g\left(\mathbf{a} \mid s^{\prime}\right)$ is a column vector of Green's functions linking all points on a to the continuous force distribution on $s$ and $g\left(s \mid s^{\prime}\right)$ is the Green's function on the interface. Equations (13)-(16) are equivalent to Eqs. (1)-(4). Following the same steps as in Eqs. (5)-(8) yields first

$$
\int_{s^{\prime}} g\left(s \mid s^{\prime}\right) f\left(s^{\prime}\right)^{\left(b_{i}\right)} d s^{\prime}=g\left(s \mid \mathbf{b}^{\mathbf{T}}\right) \mathbf{Y}_{\mathbf{a b}}^{-1} \int_{s^{\prime}} g\left(\mathbf{a} \mid s^{\prime}\right) f\left(s^{\prime}\right)^{\left({ }^{\prime} b_{i}\right)} d s^{\prime},
$$

which is equivalent to Eq. (6). A departure from the previous derivation is now required in order to discretize the

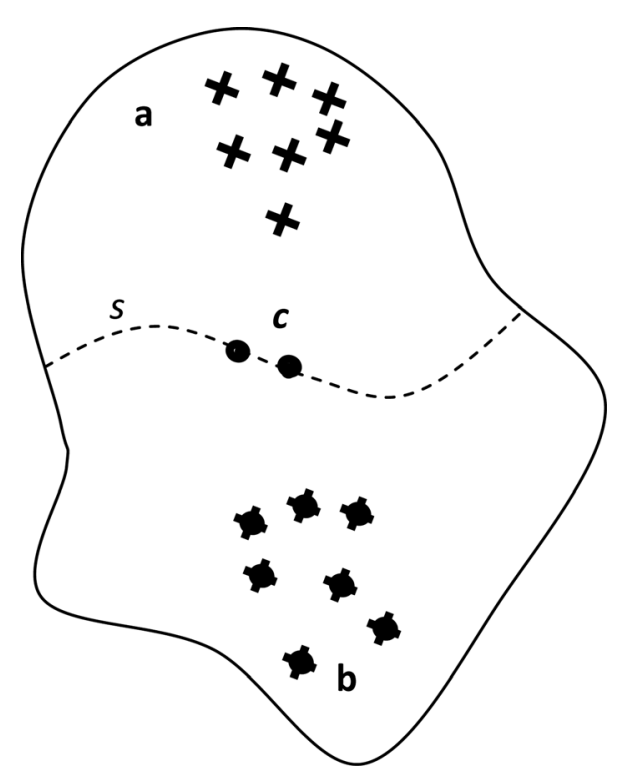

FIG. 4. Continuous structure with an arbitrary interface passing through reconstruction points $\mathbf{c}$, showing excitation points, $\mathbf{a}$ and excitation-response points $\mathbf{b}$. 

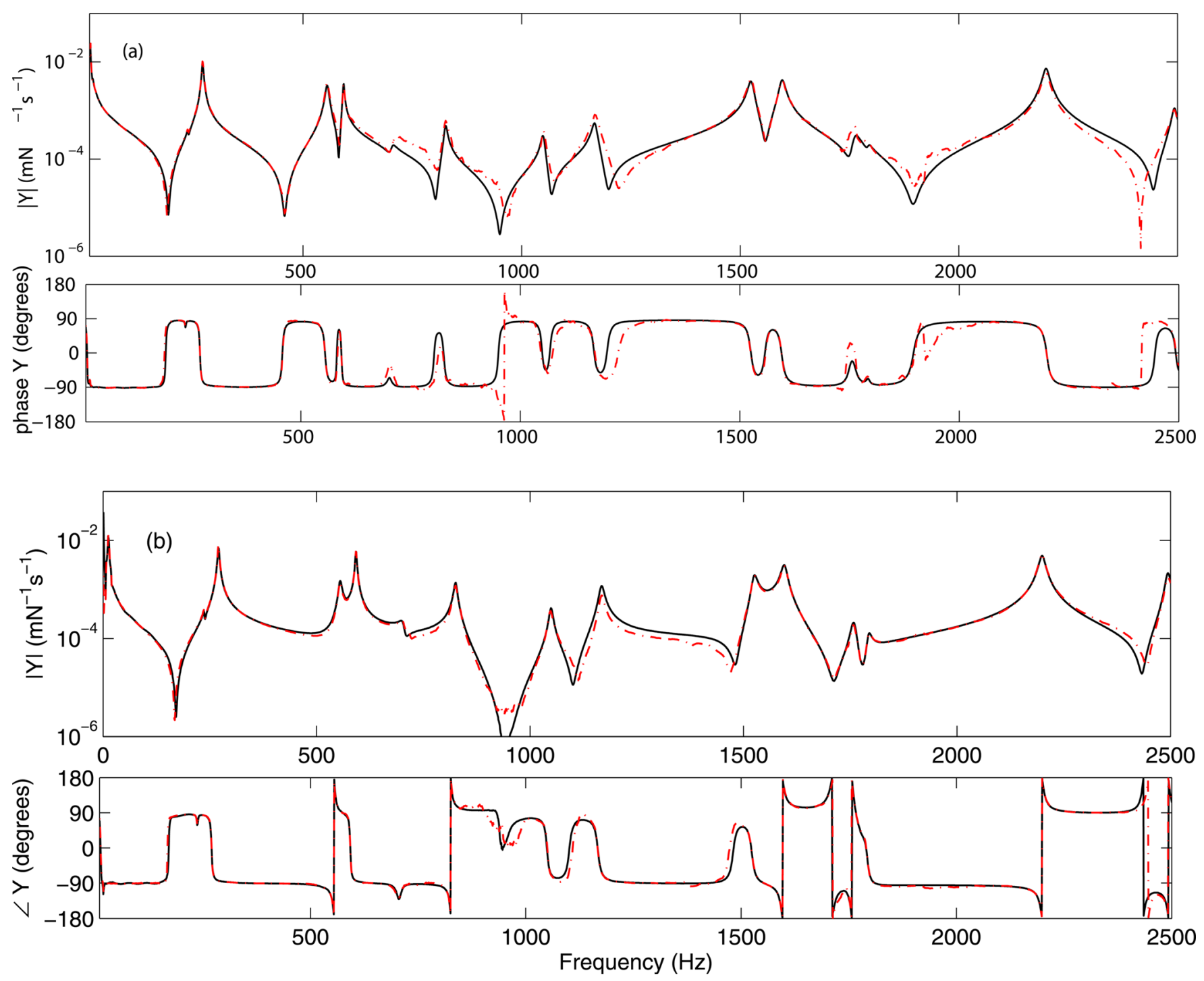

FIG. 5. (Color online) Comparison of directly measured mobility (solid line) with round trip reconstruction (dotted line) magnitude and phase at arbitrary passive points on a free aluminum plate. (a) Point mobility. (b) Transfer mobility.

continuous distributions on the interface. Thus, we approximate the force distribution as a truncated sum of $\mathrm{N}$ orthogonal basis functions $\phi_{i}(s)$ on $s$, weighted by coefficients $f_{i}$ :

$$
f(s)^{\left(b_{i}\right)} \approx \sum_{i=1}^{N} f_{i} \phi_{i}(s)=\boldsymbol{\Phi}^{\mathbf{T}}(s) \mathbf{f}^{\left(\boldsymbol{b}_{i}\right)},
$$

where in $\boldsymbol{\Phi}^{\mathbf{T}}(s)=\left\{\phi_{1}(s), \ldots, \phi_{N}(s)\right\}$ the basis functions have been arranged into a vector and $\mathbf{f}^{\left(\boldsymbol{b}_{i}\right)}=\left\{f_{1}, \ldots, f_{N}\right\}^{T}$. An example of a suitable set of basis functions would be Fourier functions, for example, as used by Bonhoff et al. ${ }^{20}$ for circular interfaces, but in principle any orthogonal set is suitable. Equation (17) is now transformed into basis function coordinates by substituting in Eq. (18), pre-multiplying both sides by $\boldsymbol{\Phi}$ and integrating over the interface response coordinate $s$. The result is

$$
\mathbf{G}_{\mathbf{s s}} \mathbf{f}^{\left(\mathbf{b}_{\mathbf{i}}\right)}=\mathbf{G}_{\mathbf{s b}} \mathbf{Y}_{\mathrm{ab}}^{-1} \mathbf{G}_{\mathrm{ac}} \mathbf{f}^{\left(\mathbf{b}_{\mathbf{i}}\right)}
$$

where $\quad\left(\mathbf{G}_{\mathbf{a s}}\right)_{i j}=\int_{s^{\prime}} g\left(a_{i} \mid s^{\prime}\right) \phi_{j}\left(s^{\prime}\right) d s^{\prime}, \quad\left(\mathbf{G}_{\mathbf{s s}}\right)_{i j}=\int_{s} \int_{s^{\prime}} \phi_{i}^{*}(s)$ $\times g\left(s \mid s^{\prime}\right) \phi_{j}\left(s^{\prime}\right) d s^{\prime} d s, \quad\left(\mathbf{G}_{\mathbf{s b}}\right)_{i j}=\int_{s} \phi_{i}(s) g\left(s \mid b_{j}\right) d s \quad$ are the
Green's functions transformed to basis function coordinates. As before, the above process is repeated with different initial excitation positions on $\mathbf{b}$ so as to build a matrix of blocked forces on each side: $\mathbf{F}=\left\{\mathbf{f}^{\left(\mathbf{b}_{\mathbf{1}}\right)} \mathbf{f}^{\left(\mathbf{b}_{2}\right)} \ldots \mathbf{f}^{\left(\mathbf{b}_{\mathbf{n}_{\mathbf{b}}}\right)}\right\}_{N \times n_{b}}$, leading to

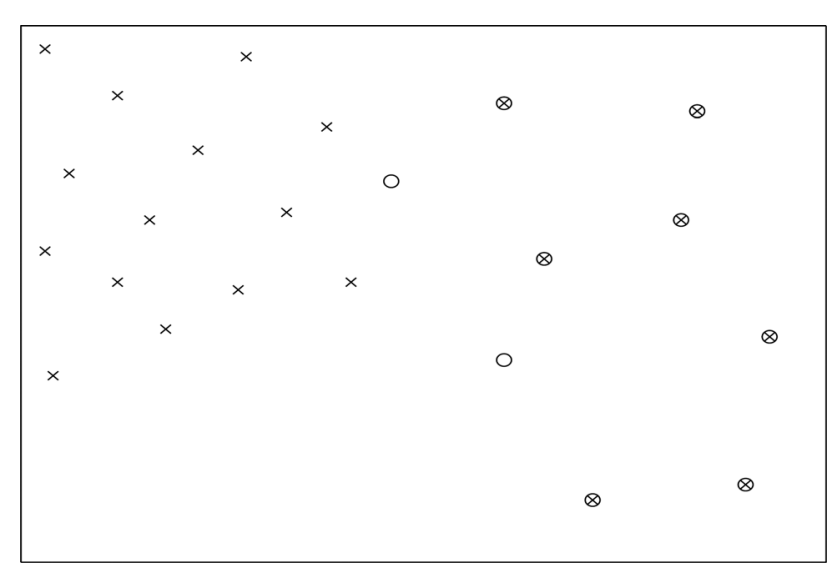

FIG. 6. Configuration of points on aluminum plate. $\bigcirc$ response measurement, $\times$ applied forces. 


$$
\mathbf{G}_{\mathrm{ss}} \mathbf{F}=\mathbf{G}_{\mathrm{sb}} \mathbf{Y}_{\mathrm{ab}}^{-1} \mathbf{G}_{\mathrm{as}} \mathbf{F}
$$

Following the same approach as led to Eq. (8), the blocked forces may be eliminated by post multiply both sides of Eq. (20) by the inverse of $\mathbf{F}$ so to obtain an identity between the transformed Green's functions. A condition for the uniqueness of the identity is $n_{b} \geq N$. Transforming back to spatial coordinates yields

$$
g\left(s \mid s^{\prime}\right) \approx g\left(s \mid \mathbf{b}^{\mathbf{T}}\right) \mathbf{Y}_{\mathbf{a b}}^{-\mathbf{1}} g\left(\mathbf{a} \mid s^{\prime}\right)
$$

valid for $n_{a} \geq n_{b} \geq N$, which is equivalent to Eq. (8). Equation (21) is valid for any excitation and response points on $s$ and since our particular interest is in points $\mathbf{c}$ it can be recast in terms of point to point Green's functions and is then effectively the same as Eq. (8),

$$
\mathbf{Y}_{\mathrm{cc}} \approx \mathbf{Y}_{\mathrm{cb}} \mathbf{Y}_{\mathrm{ab}}^{-1} \mathbf{Y}_{\mathrm{ac}}
$$

Thus, the main result of this section is to show that the round trip relation applies approximately to an arbitrary set of passive points. In what follows, this relationship will be tested experimentally.

\section{Experimental example}

Results of an experimental application of the round trip method are shown in Fig. 5. The test structure was a
$350 \mathrm{~mm} \times 500 \mathrm{~mm} \times 10 \mathrm{~mm}$ thick aluminum plate, Fig. 6 , supported on foam pads at each end. The plate was excited with a plastic tipped hammer. Seven b points and fourteen a points were randomly located either side of two reconstruction (c) points. Thus, nine accelerometers were required to measure the responses at the reconstruction and $\mathbf{b}$ points simultaneously (the a points were "excitation only" points). In order to reduce the effects of noise the solution was regularized by discarding two of the seven singular values of the $\mathbf{Y}_{\mathbf{a b}}$ matrix at every frequency. The directly measured and reconstructed results are shown for both the point Green's function, Fig. 5(a) and transfer Green's function, Fig. 5(b). It should be noted that although simple in form, the plate is experimentally an extremely challenging structure with low damping and a correspondingly high dynamic range. Moreover, the frequency range is wide for structural measurements of this type. Given these factors, the reconstructed results are convincing, extending to $2.5 \mathrm{kHz}$ and giving remarkably good accuracy at the lowest anti-resonance frequencies. The slightly less good agreement at the higher anti-resonance frequencies is perhaps an indication of the influence of the Nth order approximation since, generally, more modes contribute to the response at anti-resonances and one would expect more terms to be required in the orthogonal expansion of the force distribution along the "interface." However, further work is required to fully investigate the convergence of the algorithm.
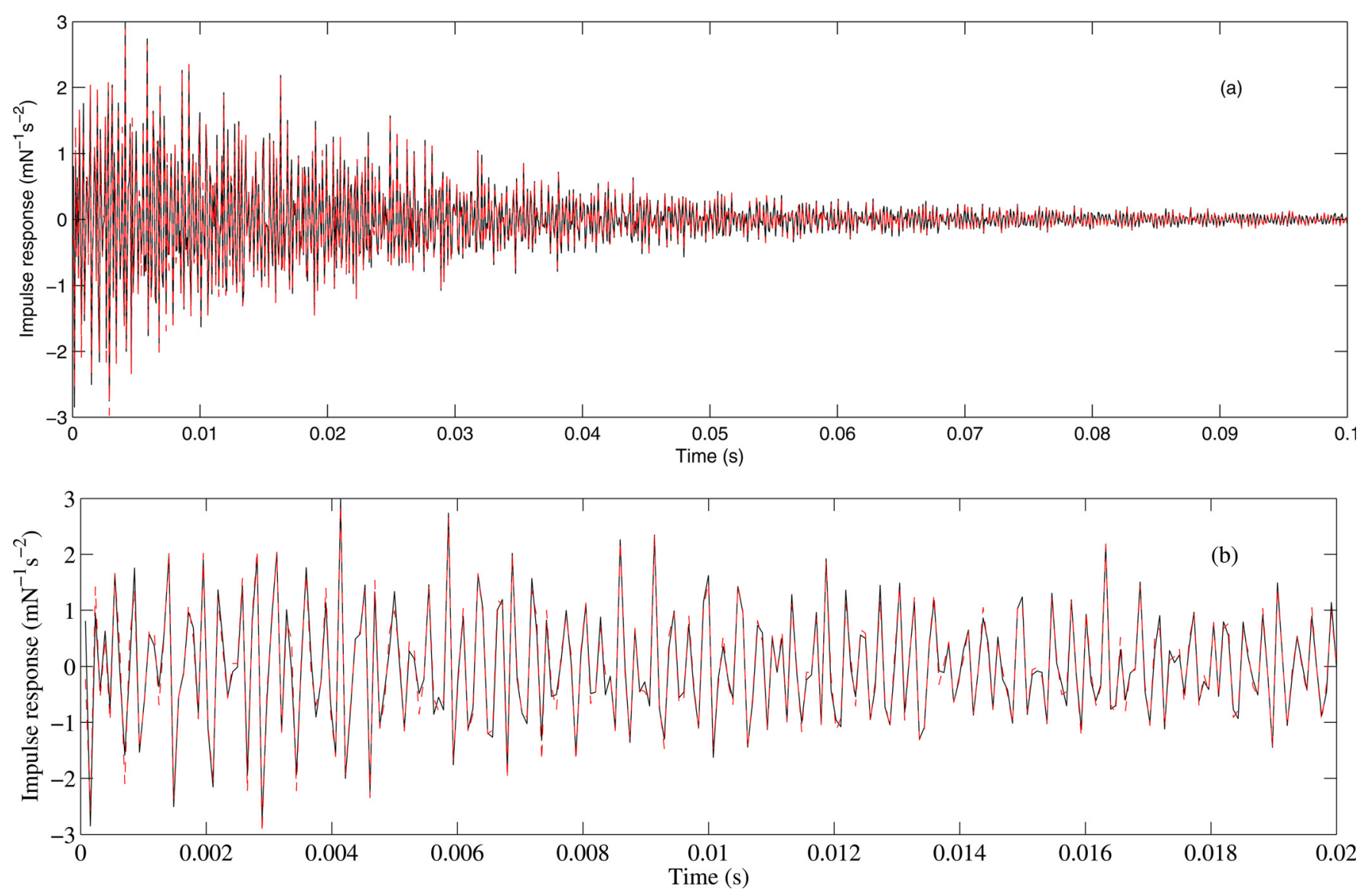

FIG. 7. (Color online) Impulse response function derived from Fig. 5(a). Directly measured (solid line), round trip reconstruction (dotted line). (a) Over $100 \mathrm{~ms}$, (b) zoom on first $20 \mathrm{~ms}$. 
A further point of interest is that, apart from a region around $1000 \mathrm{~Hz}$, the phase of the reconstructed point FRF lies within $\pm \pi$ (see comments in Sec. II B about minimum phase functions).

For completeness, shown in Fig. 7 is the impulse response function derived by inverse Fourier transformation of the point FRF results in Fig. 5(a). Good agreement is evident both of the overall shape and the details of the signal.

\section{CONCLUSIONS}

It was shown in Ref. 12 that the round trip theory gives an exact reconstruction of the Green's functions at passive points on a multi-point boundary between two substructures. The practical application of this theory has been tested experimentally by reconstructing structural Green's functions on an idealized but realistic and challenging laboratory structure. Close agreement has been obtained and the small discrepancies can be explained by expected errors in measured FRFs. This confirms that good accuracy can be obtained by applying the theory in an experimental context. A two stage measurement is required involving first, response measurements under the action of (potentially unknown) forces applied at one side of the interface and second, measurement of Green's functions with forces applied at the other side. Note that the round trip method allows retrieval of point Green's functions (same forcing and response points) which is not possible with correlation methods.

We have gone on to show that the Green's function reconstruction can be carried out at a subset of the points (or degrees of freedom) on the interface with no explicit knowledge of other interface points. The only condition is that the system be "determined" or "overdetermined" such that the total number of interface points (or degrees of freedom) does not exceed the number of excitation locations either side. This observation has led to the conjecture that the choice of boundary is arbitrary, other than necessarily including the reconstruction points. This has led to the main result of the paper which is to show that the round trip relationship applies approximately to arbitrary points. Thus, an Nth order reconstruction of the Green's function at arbitrary passive points can be achieved by combining Green's functions at points forming a round trip via a minimum of $\mathrm{N}$ excitation points either side of a "virtual" interface passing through the reconstruction points. Results from an experimental validation on a challenging structure have demonstrated convincing agreement over wide frequency range although further research is required to fully investigate the convergence of the algorithm. The results suggest that the round trip theory could offer a complementary approach to the correlation and deconvolution methods that have arisen over the last decade or so.

\section{ACKNOWLEDGMENT}

This work was supported by EPSRC under the IMP\&CTS project (EP/G066582/1).

${ }^{1}$ M. R. Ashory, "Correction of mass-loading effects of transducers and suspension effects in modal testing," in Proc. IMAC XVI - 16th International Modal Analysis Conference 2, 815-828 (1998).

${ }^{2}$ M. R. Ashory, "High quality modal testing methods," Ph.D. thesis, Imperial College London (1999).

${ }^{3}$ J. M. M. Silva, N. M. M. Maia, and A. M. R. Ribeiro, "Cancellation of mass-loading effects of transducers and evaluation of unmeasured frequency response functions,” J. Sound Vib. 236, 761-779 (2000).

${ }^{4}$ D. J. Ewins, "On predicting point mobility plots from measurements of other mobility parameters," J. Sound Vib. 70, 69-75 (1980).

${ }^{5}$ C. R. Farrar and G. H. James III, "System identification from ambient vibration measurements on a bridge," J. Sound Vib. 205, 1-18 (1997).

${ }^{6}$ C. Draeger and M. Fink, "One-channel time-reversal in chaotic cavities: Theoretical limits," J. Acoust. Soc. Am. 105, 611-617 (1999).

${ }^{7}$ O. I. Lobkis and R. L. Weaver, "On the emergence of the Green's function in the correlations of a diffuse field," J. Acoust. Soc. Am. 110, 3011-3017 (2001).

${ }^{8}$ E. Larose, L. Margerin, A. Derode, B. van Tiggelen, M. Campillo, N. Shapiro, A. Paul, L. Stehly, and M. Tanter, "Correlation of random wavefields: An interdisciplinary review," Geophysics 71, SI11-SI21 (2006).

${ }^{9}$ L. Margerin and H. Sato, "Generalized optical theorems for the reconstruction of Green's function of an inhomogeneous elastic medium," J. Acoust. Soc. Am. 130, 3674-3690 (2011).

${ }^{10}$ C. Draeger, J.-C. Aime, and M. Fink, "One-channel time-reversal in chaotic cavities: Experimental results,” J. Acoust. Soc. Am. 105, 618-625 (1999).

${ }^{11}$ A. Derode, E. Larose, M. Tanter, J. de Rosny, A. Tourin, M. Campillo, and M. Fink, "Recovering the Green's function from field-field correlations in an open scattering medium," J. Acoust. Soc. Am. 113, 2973-2976 (2003).

${ }^{12}$ A. T. Moorhouse, T. A. Evans, and A. S. Elliott, "Some relationships for coupled structures and their application to measurement of structural dynamic properties in situ," Mech. Syst. Sig. Process. 25, 1574-1584 (2011).

${ }^{13}$ Y. I. Bobrovnitskii, "A theorem on the representation of the field of forced vibrations of a composite elastic system," Acoust. Phys. 47, 507-510 (2001).

${ }^{14}$ A. T. Moorhouse, A. S. Elliott, and T. A. Evans, "In situ measurement of the blocked force of structure-borne sound sources," J. Sound Vib. 325, 679-685 (2009).

${ }^{15}$ A. S. Elliott and A. T. Moorhouse, "Indirect measurement of frequency response functions applied to the problem of substructure coupling," in Proc. NOVEM Noise and Vibration: Emerging Methods, Sorrento, Italy (2012).

${ }^{16}$ A. M. R. Ribeiro, J. M. M. Silva, and N. M. M. Maia, "On the generalisation of the transmissibility concept,” Mech. Syst. Sig. Process. 14, 29-35 (2000).

${ }^{17}$ N. M. M. Maia, J. M. M. Silva, and A. M. R. Ribeiro, "The transmissibility concept in multi-degree-of-freedom systems," Mech. Syst. Sig. Process. 15, 129-137 (2001).

${ }^{18}$ A. Elliott, A. Moorhouse, and G. Pavic, "Moment excitation and the measurement of moment mobilities," J. Sound Vib. 331, 2499-2519 (2012).

${ }^{19}$ P. A. Nelson and S.-H. Yoon, "Estimation of acoustic source strength by inverse methods: Part I, conditioning of the inverse problem," J. Sound Vib. 233, 639-664 (2000).

${ }^{20}$ H. A. Bonhoff and B. A. T. Petersson, "The influence of cross-order terms in interface mobilities for structure-borne sound source characterization: Plate-like structures," J. Sound Vib. 311, 473-484 (2008). 